\title{
Nonequilibrium noise in coupled phase oscillators
}

\author{
P. Reimann, ${ }^{1}$ C. Van den Broeck, ${ }^{2}$ and R. Kawai ${ }^{3}$ \\ ${ }^{1}$ Theoretische Physik I, Universität Augsburg, 86135 Augsburg, Germany \\ ${ }^{2}$ Limburgs Universitair Centrum, 3590 Diepenbeek, Belgium \\ ${ }^{3}$ University of Alabama at Birmingham, Birmingham, Alabama 35294
}

(Received 25 January 1999)

\begin{abstract}
The effect of nonequilibrium fluctuations on coupled phase oscillators is studied in a basic model. Its extremely rich behavior, including first and second order phase transitions, time-periodic phases, multiple anomalous hystereses, negative mobility, and other remarkable response properties, is unraveled by both analytical and numerical calculations. [S1063-651X(99)01712-2]
\end{abstract}

PACS number(s): 05.40.-a, 05.60.-k, 05.45.Xt, 87.10.+e

\section{INTRODUCTION AND MODEL}

Autonomous time-periodicity is one of the paradigms of nonlinear nonequilibrium systems which has been observed and studied in numerous physical, chemical, and biological systems [1]. Of particular interest are the collective properties that arise when a number of active oscillators are coupled. In this context, synchronization and entrainment have been documented in great detail [2]. The main purpose of this article is to highlight the surprising phenomena that emerge as a collective property of such assemblies of active oscillators, in particular autonomous rhythmicity, appearing spontaneously as the result of the coupling of the separate units, and a wealth of anomalous response properties.

The phenomena we have in mind can be studied and understood in detail on a simple variant of Kuramoto's model for coupled phase oscillators [2], described in terms of phase variables $\theta_{i}=\theta_{i}(t), i=1, \ldots, N$ :

$$
\dot{\theta}_{i}=\omega-\frac{K}{N} \sum_{j=1}^{N} \sin \left(\theta_{i}-\theta_{j}\right)+\xi_{i} .
$$

The first term $\omega$ is the rotation frequency, corresponding either to the eigen-frequency of the individual elements, or to the frequency in response to an external torque. The coupling term with strength $K>0$ is approximated by a sinus contribution, which can be viewed as the lowest order term in a Fourier expansion. Most of the results below refer to global coupling (all $N$ oscillators coupled to each other), but simulation results for nearest neighbor coupling on a twodimensional square-lattice will also be included. Finally, the noise terms $\xi_{i}=\xi_{i}(t)$ in (1) are assumed to be Gaussian and white with $\left\langle\xi_{i}(t)\right\rangle=0$ and

$$
\left\langle\xi_{i}(t) \xi_{j}\left(t^{\prime}\right)\right\rangle=2 D\left(\theta_{i}\right) \delta_{i j} \delta\left(t-t^{\prime}\right)
$$

Since the system is typically far from equilibrium, and since the reduction to phase variables $\theta$ requires a nonlinear transformation and elimination of other fast variables, we assume a state dependent "'noise-strength" $D(\theta)$ [3]. Keeping again only relevant lowest order terms in the Fourier expansion, we set $D(\theta)=D_{0}-D_{1} \cos \theta\left(D_{0} \geqslant D_{1} \geqslant 0\right)$. Analytic calculations are much simplified, while the relevant phenomena are enhanced, for the particular case $D_{0}=D_{1}=: Q / 2$, which is, in a sense, most different from purely equilibrium noise $\left(D_{1}=0\right)$ :

$$
D(\theta)=Q(1-\cos \theta) / 2=Q \sin ^{2}(\theta / 2) .
$$

Incidentally, for $\omega=0$ we may also look at (1) as XYspin-model [4] under far from equilibrium conditions. For instance, it may be exposed to a strong (but incoherent) electromagnetic irradiation, with the various effects of the photon-impacts (scattering, excitations of the host-crystal ions, etc.) represented by the nonequilibrium fluctuations (2). Much like in equilibrium phase transitions, our extremely simple model is expected to be of interest in many other contexts, corresponding to a "normal form" description, that subsists after the irrelevant terms have been eliminated. It has been chosen for its simplicity and analytic tractability, while the observed features appear to be quite general and robust.

\section{PHASE TRANSITIONS}

A detailed analytic study of (1)-(3) becomes possible in the mean field approximation, which is realized in the thermodynamic limit $N \rightarrow \infty$ [2]. Each oscillator $\theta_{i}=\theta$ then satisfies a mean field equation of the form:

$$
\begin{gathered}
\dot{\theta}=\omega-K(c \sin \theta-s \cos \theta)+\xi \\
c:=\langle\cos \theta\rangle, \quad s:=\langle\sin \theta\rangle .
\end{gathered}
$$

Here, $\langle f(\theta)\rangle$ denotes the average $\sum_{j=1}^{N} f\left(\theta_{j}\right) / N$ in the limit $N \rightarrow \infty$, or, equivalently [2], the average with respect to the the probability density $P(\theta, t)$ that obeys the following Fokker-Planck equation [5] equivalent to (4):

$$
\begin{aligned}
\partial_{t} P(\theta, t)= & \partial_{\theta}\{-\omega+K(c \sin \theta-s \cos \theta)+(Q / 4) \sin \theta \\
& \left.+Q \sin ^{2}(\theta / 2) \partial_{\theta}\right\} P(\theta, t)
\end{aligned}
$$

with periodic boundary conditions $P(\theta+2 \pi, t)=P(\theta, t)$ and normalization $\int_{0}^{2 \pi} d \theta P(\theta, t)=1$. As far as analytic calculations are concerned, we will focus mainly on the long time behavior, assuming that a steady state regime is reached (su- 
perscript st). From (4) and (6), one then finds for the mean angular velocity the two fundamental relations:

$$
\langle\dot{\theta}\rangle^{s t}=\omega+Q s / 4=2 \pi(\omega+K s) P^{s t}(0),
$$

where the order parameters $c$ and $s$ from (5) have now to be evaluated self-consistently with respect to the steady state solution $P^{s t}(\theta)$ of $(6)$.

For $\omega=0$, the equations of motion (1) are perfectly symmetric under $\theta \mapsto-\theta$. Yet, a spontaneous collective motion $\langle\dot{\theta}\rangle^{s t} \neq 0$ will set in when in (6) a solution $s \neq 0$ appears. To show that such a symmetry broken phase can indeed arise, we first study the existence and stability of the $s=0$ solution. In this case $(\omega=0$ and $s=0)$, the steady state solution of (6) takes the following closed analytical form:

$$
P^{s t}(\theta)=\mathcal{N}[\sin (\theta / 2)]^{-(c \beta+1)}, \quad \beta:=4 K / Q,
$$

where $\mathcal{N}$ is a normalization constant and $\beta>0$ is assumed. The resulting self-consistency equation for $c$ in (5) is found to be $c=(1+c \beta)(1-c \beta)^{-1}$, whence

$$
c=\left[-\left(1-\beta^{-1}\right) \pm \sqrt{\left(1-\beta^{-1}\right)^{2}-4 \beta^{-1}}\right] / 2 .
$$

A stability analysis shows that the solution with the minus sign in (9) is the stable one. In particular, for $\beta \rightarrow \infty$, one finds $c=-1$ and $P^{s t}(\theta)=\delta(\theta-\pi)$, i.e., in this strong coupling limit all the oscillators congregate at the center $\theta=\pi$ of the interval $[0,2 \pi]$. With decreasing $\beta$, the stable solution $c$ from (9) increases and disappears through a collision with the unstable one (i.e., through a limit point bifurcation) when the root in (9) becomes imaginary, that is, at the critical values:

$$
\beta_{c}=3+\sqrt{8} \approx 5.83, \quad c_{c}=1-\sqrt{2} \approx-0.41 .
$$

We conclude that only for $\beta \geqslant \beta_{c}$ there exists a solution with $s=0$ and thus $\langle\dot{\theta}\rangle^{s t}=0$. The exponent $-(c \beta+1)$ in (8) equals $\sqrt{2} \approx 1.41$ at criticality and increases with increasing $\beta$. Especially, $P^{s t}(0)=0$ for all $\beta \geqslant \beta_{c}$. For $0<\beta<\beta_{c}$ a solution with $s=0$ is excluded, implying spontaneous symmetry breaking and a spontaneous collective rotation $\langle\dot{\theta}\rangle^{s t}$ $=0$. Similar phases have been predicted in [6-9], but the quite remarkable property that the broken symmetry persists for arbitrarily small couplings $K$ and/or arbitrarily strong noise $Q$ has never been observed before.

To complement the picture, we first note that in the limit $\beta \rightarrow 0$ Eq. (6) implies $P^{s t}(\theta) \rightarrow \delta(\theta)$ and thus $s \rightarrow 0$ and $c$ $\rightarrow 1$, reflecting the fact that $\theta=0$ (modulo $2 \pi$ ) is an absorbing state for the uncoupled oscillators $[K=\omega=0$ in (4)]. Since there is no stable $s=0$ solution for small (but finite) $\beta$ values, one expects the appearance of a $s \neq 0, c \approx 1$ phase in this parameter region. Second, for $s \neq 0, \omega=0 \mathrm{Eq} .(7) \mathrm{im}-$ plies $P^{s t}(0)=(2 \pi \beta)^{-1}$. Recalling that $P^{s t}(0)=0$ for $s=\omega$

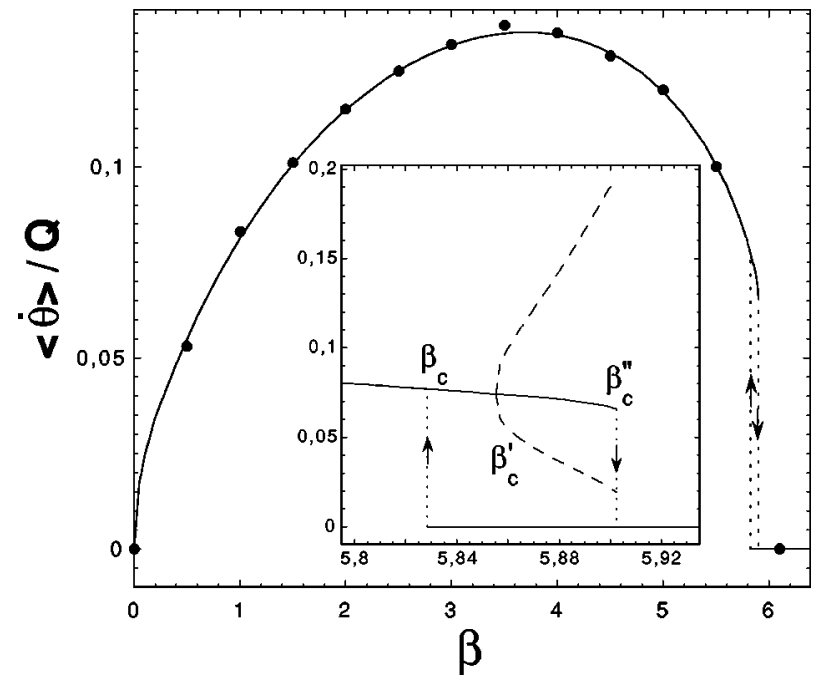

FIG. 1. Steady state frequency $\langle\dot{\theta}\rangle^{s t}$ as a function of $\beta$ $=4 K / Q$ for the dynamics $(1-3)$ with $\omega=0$. Solid: $N \rightarrow \infty$ by solving (10). Dots: illustrative verification by direct simulations of (1) for $N=1024$. Coexisting negative solutions $\langle\dot{\theta}\rangle \mapsto-\langle\dot{\theta}\rangle$ are omitted. Inset: Details of the hysteresis. For $\beta_{c}^{\prime}<\beta<\beta_{c}^{\prime \prime}$ a $\langle\dot{\theta}\rangle^{s t}=0$ phase coexists with a time-periodic solution. There, the solid line represents the time-averaged $\langle\dot{\theta}\rangle$. Dashed: Extrema of the oscillating $\langle\dot{\theta}\rangle$. Dotted with arrows: Jumps of $\langle\dot{\theta}\rangle$.

$=0$ we conclude that the phase $s \neq 0$ must appear through an abrupt change in $P^{s t}(\theta)$, i.e., a discontinuous transition. An unfortunate consequence is that a perturbation analysis will fail, hence an analytic treatment of this phase appears to be difficult. We therefore finally turn to the numerical results collected in Fig. 1 [10]. In addition to our theoretical predictions, one finds that in the region $0<\beta<\beta_{c}^{\prime}$, with $\beta_{c}^{\prime}$ $\approx 5.86$ a $s \neq 0$ solution exists, which is (numerically) stable, stationary, and-apart from the reflection-symmetry induced degeneracy-unique. At $\beta_{c}^{\prime}$ it loses its stability by a Hopf bifurcation with the quite unexpected appearance of a timeoscillatory behavior of $\langle\dot{\theta}\rangle$. Finally, at another critical value $\beta_{c}^{\prime \prime} \approx 5.90$, the stable limit cycle suddenly disappears (presumably through a collision with an unstable limit cycle). The observed coexistence of stable solutions in the region $\beta_{c}<\beta<\beta_{c}^{\prime \prime}$ implies hysteresis, with, upon increasing $\beta$, a discontinuous (first order) transition from the oscillatory $s$ $\neq 0$ solution to $s=0$ at $\beta=\beta_{c}^{\prime \prime}$, and upon subsequently decreasing $\beta$, a discontinuous transition from the $s=0$ solution back to a steady state solution $s \neq 0$ at $\beta=\beta_{c}$. Spontaneous collective oscillations have been long known $[1,2,6,11,12]$. The distinguishing features of our model (1) with $\omega=0$ are its extreme simplicity and its symmetry under reflection and discrete translation.

\section{RESPONSE PROPERTIES}

Returning to (7), we note another remarkable feature with surprising consequences: since $\operatorname{sign}\left(\langle\dot{\theta}\rangle^{s t}\right)=\operatorname{sign}(s)$ (Eq. (7) with $\omega=0)$, it follows that the direction of the spontaneous rotation is opposite to that of the symmetry breaking. For example, for $s>0$ one expects from (5) a majority of the oscillators in the "left" part of the interval $[0,2 \pi]$, while 

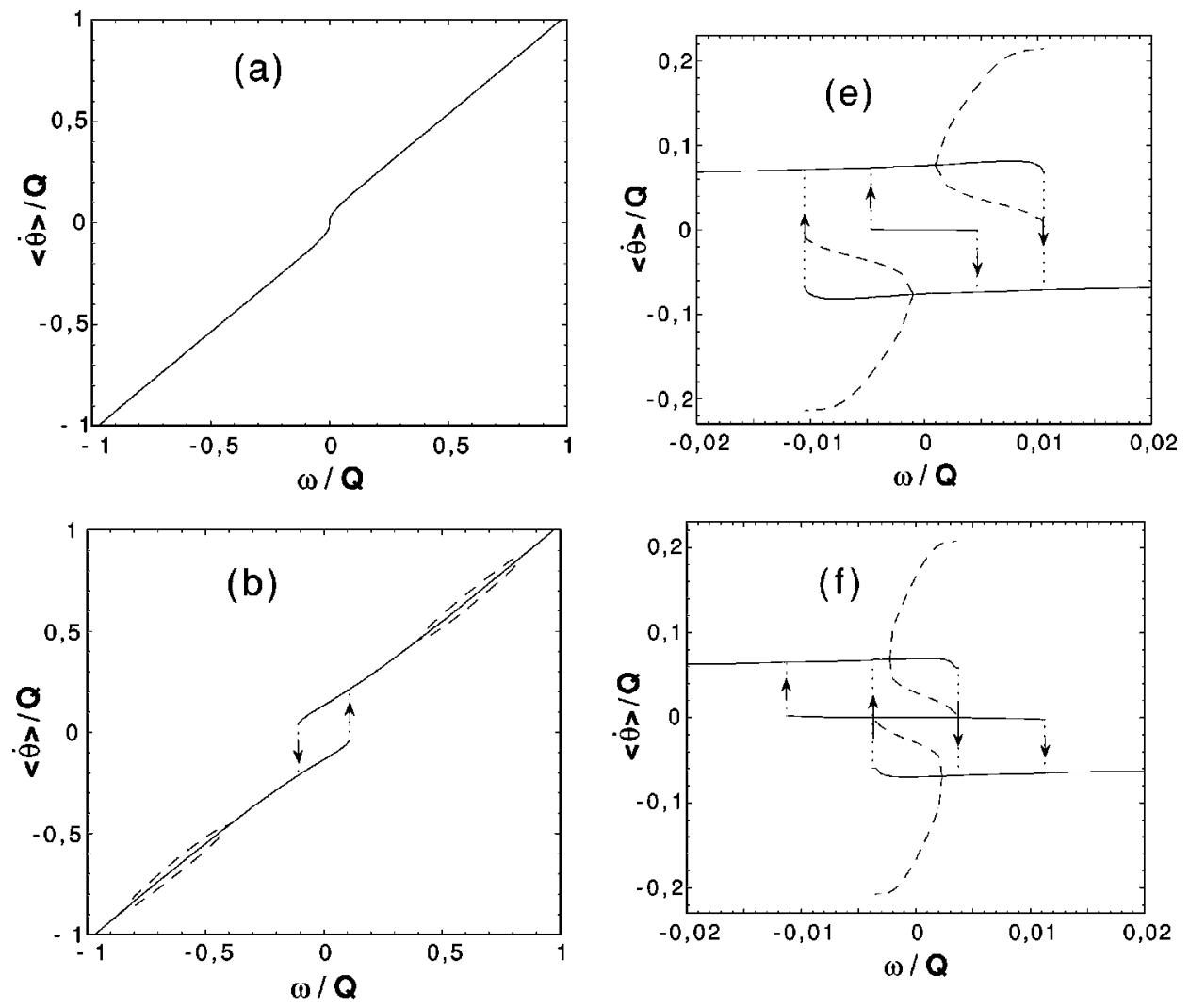

FIG. 2. Scaled response curves [8] $\langle\dot{\theta}\rangle / Q$ versus $\omega / Q$ for $\beta=0$ (a), $\beta=3.4$ (b), $\beta=5.14$ (c), $\beta$ $=5.16(\mathrm{~d}), \beta=5.84$ (e), $\beta=5.89$ (f), $\beta=5.92(\mathrm{~g}), \beta=6.5$ (h). Solid and dashed lines are analogous to Fig. 1. For more details see main text.
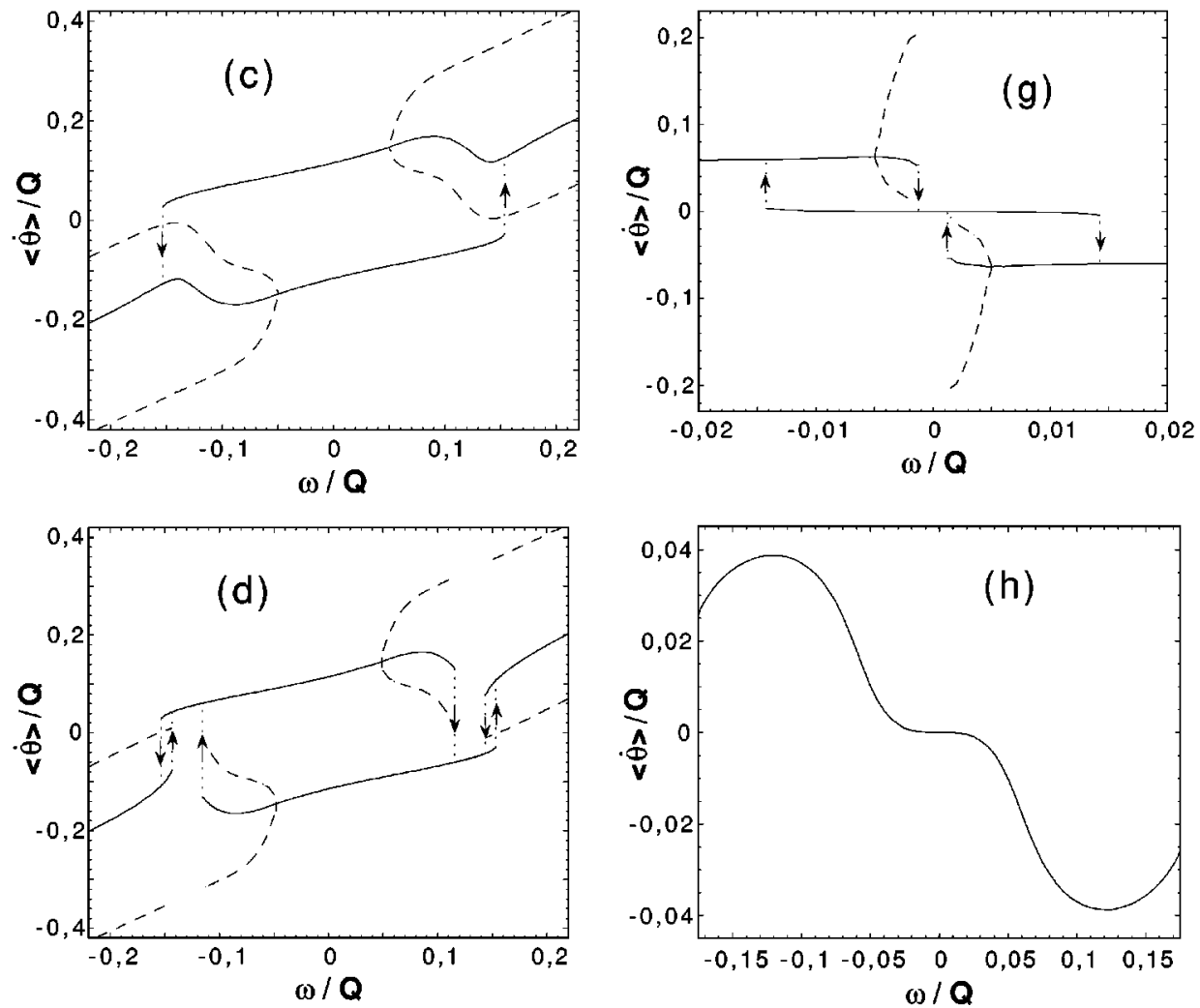

$\langle\dot{\theta}\rangle^{s t}>0$ implies that there is a net rotation towards the "right." This raises the question as to whether an applied torque that concentrates $P^{s t}(\theta)$ on one side of the interval can in fact result in a overall rotation of the oscillators in the other direction. The following simple argument shows that for sufficiently large $\beta$ this is indeed the case. In the limit of strong coupling, $\beta \rightarrow \infty$, one expects that $P^{s t}(\theta)$ becomes sharply peaked around a central value $\bar{\theta}, \quad P^{s t}(\theta) \rightarrow \delta(\theta$ $-\bar{\theta}$ ). If furthermore $|\omega|<Q / 4$ then it follows from Eqs. (5) and (7) that the flux $\langle\dot{\theta}\rangle^{s t}=\dot{\bar{\theta}}=\omega+Q \sin \bar{\theta} / 4$ approaches zero in the steady state, so $s=\sin \bar{\theta}=-4 \omega / Q$. The symmetry breaking is thus, as expected, in the same direction as $\omega$ (e.g., $\bar{\theta}>\pi$ for $\omega>0$ ). Inserting this $\beta \rightarrow \infty$ result for $s$ in the 
second equality in (7), we conclude that for large but finite $\beta$

$$
\langle\dot{\theta}\rangle^{s t}=-2 \pi \beta P^{s t}(0) \omega
$$

implying [13] absolute (in contrast to differential) negative mobility [8]. Note that this it is not the result of a built-in asymmetry; the oscillators are always rotating opposite to the torque, irrespective of its direction. In other words, the coupling and noise in (1) may indeed conspire such that a collective rotation just opposite to the inborn-frequency $\omega$ of the separate oscillators emerges!

The analytically not tractable response behavior for smaller $\beta$ is an intricate story in itself and we can only briefly sketch here the numerically observed wealth of bifurcations. The uncoupled case $\beta=0$ [Fig. 2(a)] is unspectacular with the exception of the square-root behavior about $\omega$ $=0$. The latter turns into a normal hysteresis-loop (like in a ferromagnet) as soon as $\beta>0$, see Fig. 2(b). The hysteresisloop grows and at $\beta \approx 3.33$ a re-entrant Hopf-bifurcation into an oscillatory long-time behavior of $\langle\dot{\theta}\rangle$ takes place [Fig. 2(b)]. Upon a further increase of $\beta$, the re-entrance points of these oscillations move away to infinity, and the oscillations then persist for arbitrary large values of $|\omega|$. Upon increasing $\beta$ further, the Hopf-bifurcation points penetrate the hysteresis loop [Fig. 2(c)]. One of the branches develops a "dip" [Fig. 2(c)] which at $\beta \approx 5.15$ touches the other branch and then gives rise to a non-oscillatory gap [Fig. 2(d)], with, at the outside end of the gap, the appearance of a new "small" hysteresis loop. The latter shrinks and finally disappears at $\beta \approx 5$.26. In its place an infinite-period bifurcation remains [omitted in Figs. 2(e)-2(h) in favor of the small $\omega$-region], together with the "large" hysteresis from Fig. 2(d). In comparison with the usual case in Fig. 2(b), this hysteresis-loop is anomalous [8] in that the direction of the discontinuous jumps of $\langle\dot{\theta}\rangle$ are just opposite to the applied torque $\omega$. At $\beta=\beta_{c} \approx 5.83$ a new stable branch arises inside the anomalous hysteresis-loop [Fig. 2(e)], in agreement with Eq. (10). It quickly grows beyond the loop [Fig. 2(f)], while the loop shrinks to zero at $\beta=\beta_{c}^{\prime \prime} \approx 5.90$, leaving behind a symmetric pair of anomalous loops [Fig. 2(g)] together with a small region of negative absolute mobility about $\omega=0$. The hysteresis-loops subsequently shrink and disappear at $\beta$ $\approx 6.05$, resulting in an extended zone of absolute negative mobility [Fig. 2(h)] as predicted by Eq. (11). With further increasing $\beta$ the absolute values of $\langle\dot{\theta}\rangle / Q$ decrease but the negative mobility around $\omega=0$ subsists as well as the infinite period bifurcation into an oscillatory solution for large $\omega$, completing our picture of the surprisingly rich response behavior contained in the simple model (1).

\section{GENERALIZATIONS AND CONCLUSION}

All the results so far refer to global coupling in Eq. (1). The extension to finite range interactions is clearly of central importance but very difficult to handle analytically. Numerically, a similar behavior as for global coupling is observed for nearest neighbor interaction on, for example, a square lattice in $d=2$, cf. Fig. 3. In particular, the salient features are clearly reproduced, namely a spontaneous symmetry breaking for $\omega=0$ and moderate-to-weak coupling, con-
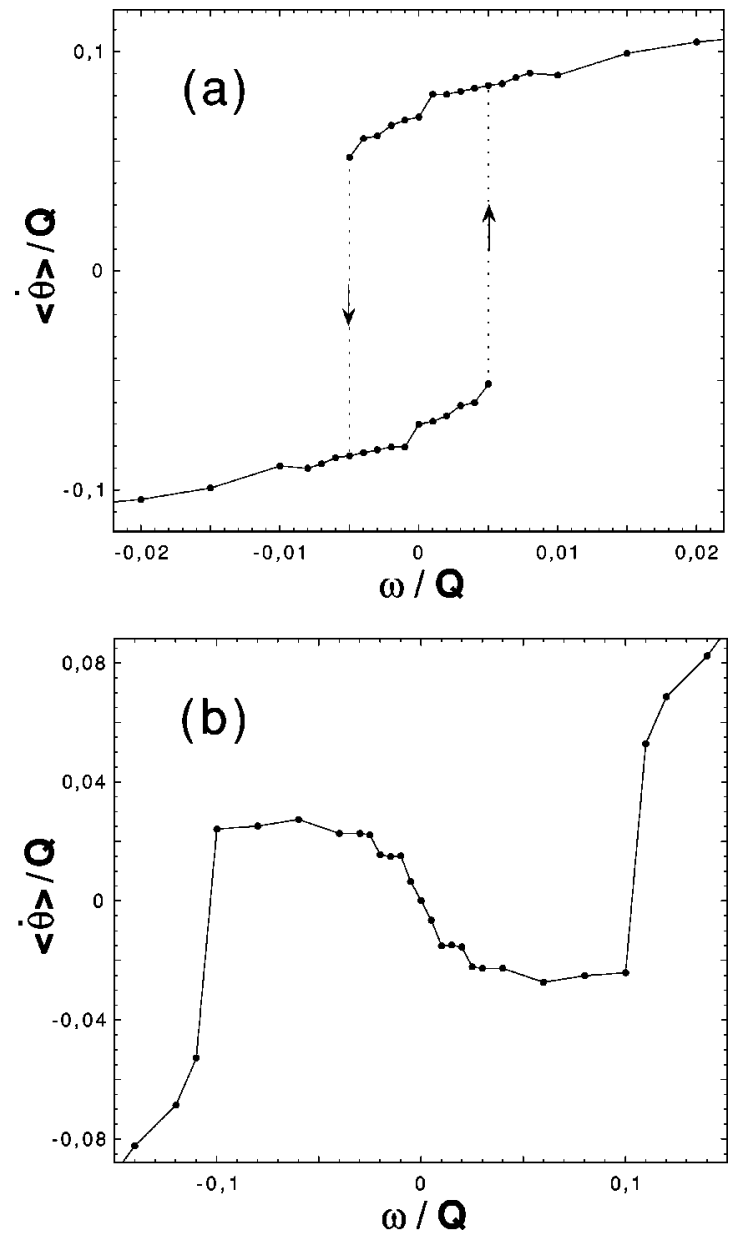

FIG. 3. Scaled response curves [8] $\langle\dot{\theta}\rangle / Q$ versus $\omega / Q$ from numerical simulations of (1-3) with nearest neighbor instead of global coupling in (1) for a $64 \times 64$ square-lattice with periodic boundary conditions (see [7] for more computational details). (a): Example of spontaneous collective rotation (at $\omega=0)$ and hysteresis for $\beta=3$. (b): Absolute negative mobility for $\beta=8$. While those qualitative features are the same as for global coupling, the quantitative details are different.

nected with a hysteresis-loop in response to finite $\omega$ [Fig. $3(\mathrm{a})$ ], as well as absolute negative mobility for stronger coupling [Fig. 3(b)]. We conclude that those phenomena are quite robust.

In summary, our model (1) with $\omega=0$ provides a extremely simple example of a pure noise-induced phase transition [9] with-for the first time-a fully analytically tractable symmetric phase (Eqs. (8)-(10)). In particular, no deterministic external forces are involved and-due to the zeros of the noise amplitude (3) - we are essentially dealing with a zero temperature model (no additive noise component). It therefore seems a good candidate for becoming a prototype model for phase transitions induced by nonequilibrium fluctuations. Also, to the best of our knowledge, its most striking features have not been observed before in such a context, namely (see Fig. 1) a spontaneously broken symmetry for arbitrarily weak coupling and a discontinuous transition with a permanently time-dependent oscillatory phase. While spontaneous symmetry breaking is a familiar phenomenon also at equilibrium, spontaneous collective rotations would contradict the second law of thermodynamics and are 
therefore a distinct nonequilibrium feature. The response behavior of our model (1) in the presence of a torque $\omega$ comprises a whole wealth of interesting novel phenomena (Fig. 2) together with some previously observed effects in coupled phase oscillators [11] and other models of collective phenomena $[6-8,12]$. The particular appeal of our system is its archetypal simplicity, analytic tractability, and extreme richness in behavior.

\section{ACKNOWLEDGMENTS}

This work was supported by DFG-Sachbeihilfe HA1517/ 13-2, the Graduiertenkolleg GRK283, the Program on InterUniversity Attraction Poles of the Belgian Government, the F.W.O. Vlaanderen, NATO Grant No. CRG 950055, the Alabama Education and Research Network, and the Alabama Supercomputer Center.
[1] G. Nicolis and I. Prigogine, Self Organization in Nonequilibrium Systems, edited by C. Vidal and A. Pacault (Springer, Berlin, 1981); Nonequilibrium Dynamics in Chemical Systems, ibid 1981; A.T. Winfree, The Geometry of Biological Time (Springer, Berlin, 1980); L. Glass and M.C. Mackey, From Clocks to Chaos (Princeton University Press, Princeton, NJ, 1988).

[2] Y. Kuramoto, Chemical Oscillations, Waves, and Turbulence (Springer, Berlin, 1984); S.H. Strogatz in Frontiers in Mathematical Biology, edited by S. Levine (Springer, Berlin, 1994), and further references therein.

[3] Since the unperturbed frequencies $\omega$ are not required to be large in comparison with the interaction and the noise in (1), a transformation to an effective state-independent noise by "phase-averaging" [2] is not possible in our case. We further note that the factor $2 D\left(\theta_{i}\right)$ in (2) can equivalently be absorbed as "multiplicative noise" $\sqrt{2 D\left(\theta_{i}\right)} \xi_{i}$ in (1). The latter is to be understood in the physically relevant sense of Stratonovich [5].

[4] A. Arenas and C. Vicente, Phys. Rev. E 50, 949 (1994).
[5] H. Risken, The Fokker-Planck Equation (Springer, Berlin, 1984).

[6] F. Jülicher and J. Prost, Phys. Rev. Lett. 75, 2618 (1995); F. Jülicher, A. Ajdari, and J. Prost, Rev. Mod. Phys. 69, 1269 (1997).

[7] K.A. Alekseev et al., Phys. Rev. Lett. 80, 2669 (1998).

[8] P. Reimann, R. Kawai, C. Van den Broeck, and P. Hänggi, Europhys. Lett. 45, 545 (1999).

[9] C. Van den Broeck, J.M.R. Parrondo, R. Toral, and R. Kawai, Phys. Rev. E 55, 4084 (1997).

[10] Note that $Q$ can be absorbed into the time-scale in (1), thus $\langle\dot{\theta}\rangle / Q$ depends only on $\beta=4 K / Q$ and $\omega / Q$.

[11] S. Shinomoto and Y. Kuramoto, Prog. Theor. Phys. 75, 1105 (1986); J.A. Acebron, L.L. Bonilla, S. De Leo, and R. Spigler, Phys. Rev. E 57, 5287 (1998).

[12] H. Gang, H. Haken, and X. Fagen, Phys. Rev. Lett. 77, 1925 (1996).

[13] For $\omega \neq 0$ it can be shown rigorously that $P^{s t}(0)>0$. 